\title{
Insulin Pump Best Practice Guide from the ABCD Diabetes Technology Network UK
}

\author{
EMMA G WILMOT, ${ }^{1}$ PETER HAMMOND, ${ }^{2}$ PRATIK CHOUDHARY ${ }^{3}$
}

\begin{abstract}
The number of children and adults with Type 1 diabetes on insulin pump therapy in the UK continues to rise. However, there remains significant geographical variation in uptake. It is likely that health care professional training is a key factor driving this variation. In response, the Diabetes Technology Network UK has developed a national best practice guide to support health care professionals to develop and maintain their skills in insulin pump therapy. An overview of this guide is provided
\end{abstract}

Br J Diabetes 2018;18:69-70

Key words: insulin pump, type 1 diabetes, continuous subcutaneous insulin infusion (CSII), best practice, Diabetes Technology Network

Since NICE published the technology appraisal for insulin pump therapy in 2008, the number of people living with type 1 diabetes who have been able to access this technology has steadily increased. More and more centres are starting to use insulin pump therapy, although there still remains significant variation in access across the four nations. In addition to this, the Diabetes Technology Network has been aware of a large degree of variation in practice across the country, and at our last two national meetings there was a strong call for a guide that could summarise current evidence and experience to allow best practice to support clinicians in all areas of the country.

Over the past year a large group of clinicians with expertise in insulin pump therapy have been working together to develop the Diabetes Technology Network UK Best Practice Guide: 'Continuous Subcutaneous Insulin Infusion (CSII), A Clinical Guide for Adult Diabetes Services'. This document was launched at the Diabetes UK annual professional conference in March 2018 where 100 printed copies flew off the shelves. It is hoped that the best practice guide will help to standardise clinical care across the four nations and support centres keen to upskill their team members on CSII, reducing variation in care.

Key topics covered in this guide include recommendations for insulin pump initiation, with a useful summary chart of the advan-

Derby Teaching Hospitals NHS Foundation Trust, Derby, UK

2 Harrogate and District Foundation Trust, Harrogate, UK

${ }^{3}$ King's College London, London, UK

Address for correspondence: Dr Emma Wilmot

Derby Teaching Hospitals NHS Foundation Trust, Derby, DE22 3NE, UK

E-mail:emma.g.wilmot@gmail.com

https://doi.org/10.15277/bjd.2018.176 tages and disadvantages which clinicians might find useful to share in consultations with those contemplating pump therapy. The benefits of insulin pump therapy can include improvement in glucose control and quality of life as well as a reduction in hypoglycaemia. However, the guide does not neglect the downside, with a focus on the data from John Pickup highlighting the sometimes frequent occurrence of insulin pump set kinking or blockage. Selection criteria for those most likely to benefit are discussed with a flow chart which highlights the need to consider additional aspects such as psychological morbidity, excluding underlying coeliac disease and considering whether the user has retinopathy and might require ophthalmologist review prior to initiation.

Starting out on the right foot with pump therapy is really important and, as such, a chart used at a number of large centres that helps calculate suggested initial CSII settings is included alongside a discussion on how to further optimise settings. A modified basal profile was the group's preferred basal profile of choice over a circadian profile. Similarly, although many units have used the 500/100 rules, recent publications and experience within the group led to the recommendation of a starting insulin to carbohydrate ratio of 400/total daily dose and an insulin sensitivity factor of 130/total daily dose. A useful 'at a glance' table is provided to help with these calculations.

The best practice guide includes a review and scenarios of where advanced insulin pump features such as temporary basal rates and multi-wave boluses may be used. For instance, temporary basal rates may be utilised in some scenarios that increase (eg, stress, illness) or decrease (eg, physical activity, alcohol) insulin requirements. The latest evidence indicates that dual wave boluses with high fat/protein meals may require a $25-30 \%$ increase in the calculated bolus dose, delivered as a dual wave bolus with $50-70 \%$ up front and the rest delivered over 2-6 hours in an attempt to prevent late postprandial hyperglycaemia.

Throughout the guide there is a focus on the need for pump centres to have access to CSII downloads which inform optimisation strategies. A table outlining a potential approach to download interpretation is included. In particular the glucose targets in CSII are discussed, highlighting the fact that different pump models correct high glucose levels in different ways. Some pumps correct to the higher value in the target range while others correct to the mid point of the target range. To minimise confusion with this, we recommend a narrow target range (4.5-5.5 mmol/L) across all devices that helps provide a consistent simple message to clinicians and insulin pump users.

It is known that infusion set kinking and blockages do occur and, as such, there is a dedicated section on the management of unexplained hyperglycaemia and also sick day rules. We have also referenced a published pathway developed by an international 


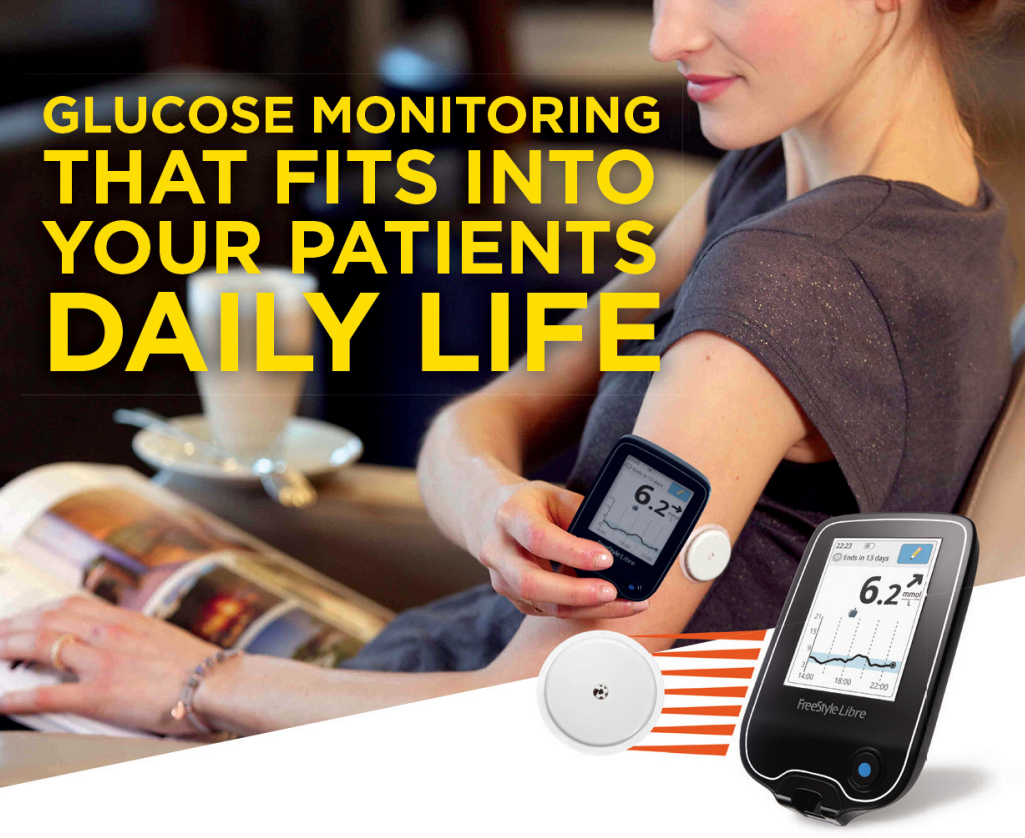

\section{Digital health tools that work together} for seamless diabetes management

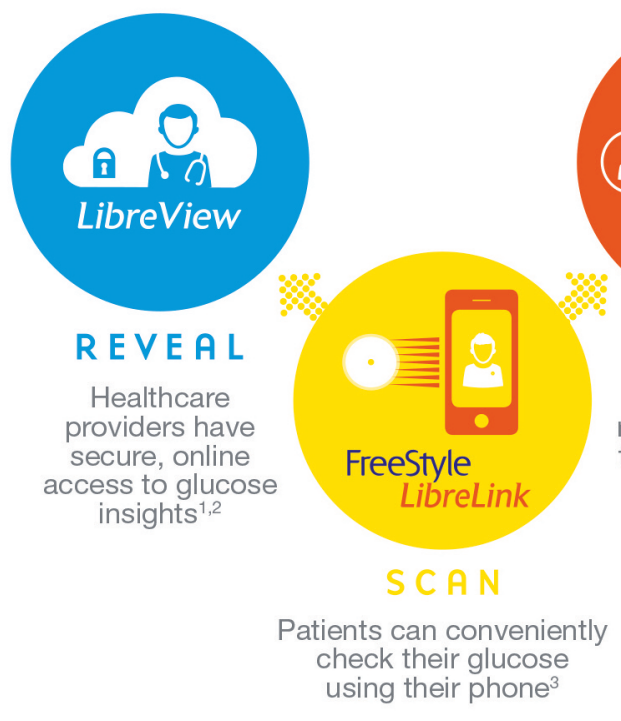

Find out more at www.FreeStyleDiabetes.co.uk

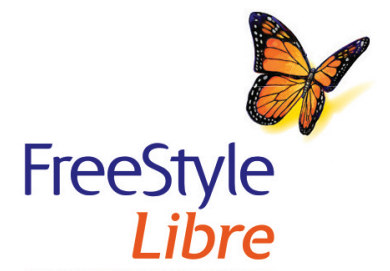

FLASH GLUCOSE MONITORING SYSTEM

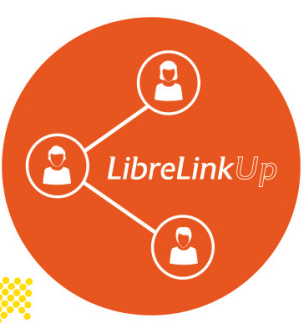

C ONNECT

Caregivers can

remotely monitor their loved ones ${ }^{4}$ using their phone ${ }^{3}$

1. LibreView data can be viewed in the Safari Browser on Mac OS X Mountain Lion or higher computers and on iOS 6 or higher mobile devices. Currently, uploading of glucose data is only
supported on Windows-based computers. Minimum system requirements are Windows Vista with IE10 or the latest versions of Google Chrome and Mozilla Firefox, running on a 550MHz Pentium III, 512MB DRAM, 2G Hard Drive, USB 2.0, LCD Screen with resolution of 1024x768. 2. LibreView (including the LibreLinkUp mobile app) is not intended to be a primary glucose monitor: home users must consult their primary device(s) and consult a healthcare professional before making any medical interpretation and therapy adjustments from the information in the software, and healthcare professionals should use information in the software in conjunction with other clinical information available to them. 3. The FreeStyle LibreLink app is compatible with NFC enabled phones running Android OS 5.0 or higher and with iPhone 7 and higher running OS 11 and higher. 4. LibreLinkUp is a mobile application, developed and provided by Newyu, Inc. Use of LibreLinkUp
requires registration with LibreView, a service provided by Abbott and Newyu, Inc.

FreeStyle, Libre and related brand marks are trademarks of Abbott Diabetes Care, Inc. in various jurisdictions. Date of preparation: May 2018. ADCMDP180062

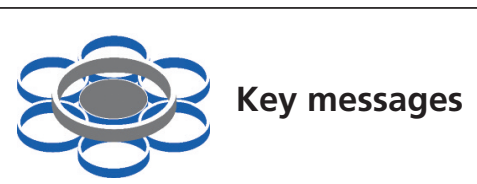

- There is considerable variation in access and outcomes of pump therapy

- Collating best practice in a national document may reduce variation in practice and improve outcomes

- The full guide is available via the ABCD Diabetes Technology Network website:

https://abcd.care/dtn-uk-best-practice-guides

consensus group on the appropriate hierarchy of use of different technologies in those with problematic hypoglycemia. The guide has examples of validated tools such as the Clark and Gold scores used to assess awareness of hypoglycaemia.

A contentious issue in CSII therapy is when to consider discontinuation. In particular, it is important to consider the safety aspects of people who are using CSII but performing inadequate blood glucose monitoring. This is a challenging area but, on balance, the group of experts and people living with diabetes who were involved in development of the guide felt that inadequate monitoring of glucose should be an indication for withdrawal, given the potential risks of unrecognised infusion set blockage/kinking which may quickly result in ketosis and progression to diabetic ketoacidosis if the glucose levels are not adequately monitored. Additionally, an absence of clinical benefit or failure to attend follow-up would be deemed potential indications for discontinuation.

Alistair Lumb from Oxford contributed the section on exercise and CSII, a valuable starting point for supporting active pump users, with reference to a recently published paper with more detailed information. Various approaches are discussed for managing both aerobic and anaerobic exercise including reducing basal and bolus insulin to counteract the effects of exercise and prevent hypoglycaemia. A 20\% reduction in insulin for 4-6 hours from going to bed is suggested to help prevent nocturnal hypoglycaemia when the exercise has occurred later in the day.

The guide is extensively referenced to provide the evidence behind the recommendations and direct the reader to more detailed guidance where needed - for example, in exercise management. Overall, the development group hope that this document serves as a useful 'go to' guide for those working in and developing skills to deliver CSII services. A copy of the Best Practice Guide can be found at www.dtn-uk.care.

Conflict of interest: Dr Wilmot reports personal fees from Abbott Diabetes Care, Diasend, Dexcom, Eli Lilly, Medtronic, Sanofi Aventis and Novo Nordisk, outside the submitted work; Dr. Hammond reports personal fees from Medtronic, Roche, Abbott, Novo Nordisk, CellNovo Sanofi, Lilly, Insulet and Diasend/Glooko. Dr. Choudhary reports personal fees from Medtronic, Roche, Novo Nordisk, AstraZeneca, Eli Lilly, Abbott Diabetes, Sanofi and Ascenscia Ltd.

\section{Funding: None}

\section{References}

1. ABCD Diabetes Technology Network CSII Best Practice Guide. Continuous subcutaneous insulin infusion (CSII). A clinical guide for adult diabetes services. https://abcd.care/dtn-uk-best-practice-guides (last accessed 16 April 2018) (all references can be found at the end of the Best Practice Guide) 